\title{
Mosca barrenadora de la madera \\ (Pantophthalmus sp.) en Hieronyma \\ alchomeoides Allemäo en Costa Rica. Primer registro
}

Marcela Arguedas-Gamboa ${ }^{1}$

María Rodríguez-Solís ${ }^{2}$

Víctor Martínez

\section{Resumen}

Se reporta por primera vez larvas de Pantophthalmus sp. (Pantophthalmidae, Diptera) barrenando la madera de árboles de Hieronyma alchoneoides, en una plantación mixta de 15 años en la zona atlántica de Costa Rica. Se describe la larva y se caracterizan los daños causados al hospedero.

Palabras clave: Especies nativas, Pantophthalmus, Hieronyma alchorneoides, Costa Rica.

\section{Abstract}

Timber borer fly (Pantophthalmus sp.) in Hyeronima alchorneoides Allemão in Costa Rica. First registration.

A record of Pantophthalmus sp. larvae. (Pantophthalmidae, Diptera) is reported for the first time boring the wood of Hieronyma alchoneoides trees in a mixed plantation of 15 years in the Atlantic zone of Costa Rica. The larva is described and the damage to the host is characterized.

Keywords: Native tree species, Pantophthalmus, Hieronyma alchorneoides, Costa Rica.
1. Instituto Tecnológico de Costa Rica, Escuela de Ingeniería Forestal; Cartago, Costa Rica; marguedas@itcr.ac.cr

2. Instituto Tecnológico de Costa Rica, Escuela de Ingeniería Forestal; Cartago, Costa Rica; maria.rodriguez@itcr.ac.cr

3. Reforest The Tropics Inc.vjmartinez89@gmail.com 


\section{Introducción}

La reforestación con especies nativas en las regiones tropicales es una opción para acelerar los procesos de recuperación de la biodiversidad en áreas degradadas (Montero, De los Santos Posada \& Kanninen, 2007). En general, la diversificación de especies en plantaciones es deseable debido a la incertidumbre sobre el desempeño de las especies y los riesgos potenciales de plagas. A su vez, económicamente las plantaciones mixtas tendrán la ventaja de diversificar la producción, que podría reducir los riesgos para los agricultores en mercados inestables. La reforestación con especies nativas en Costa Rica se ha desarrollado en torno a unas pocas especies, que presentan condiciones favorables de crecimiento y una alta demanda a nivel de mercado (Alice, Montagnini \& Montero, 2004).

En Centroamérica, el pilón (Hieronyma alchorneoides) se ha utilizado en programas de reforestación con incentivos, reforestación de claros de bosque, sistemas agroforestales y en plantaciones puras o mixtas (Montero et al., 2007). En Costa Rica, esta es una de la especies nativas maderables que debido a sus características de crecimiento y adaptabilidad, se ha ajustado a condiciones abiertas de plantación, además, gracias a la variedad de usos, la demanda por material de siembra ha aumentado (Abdelnour, Aguilar \& Valverde, 2011).

Durante el primer semestre del 2014, se informa la presencia de larvas de la "mosca barrenadora", en árboles de pilón en una plantación mixta en la zona atlántica de Costa Rica. Estas larvas pertenecen a la familia Pantophthalmidae (orden Diptera), la cual es una pequeña familia cuyas especies se encuentran únicamente en los bosques húmedos de la región neotropical (Val, 1976). En este estudio se diagnostica la especie, se describe la larva y se caracterizan los daños causados al hospedero.

\section{Material y métodos}

\section{Área de estudio}

La presencia de la plaga se reporta en la finca forestal Hacienda Las Delicias, ubicada en el cantón de Guácimo, provincia de Limón, Costa Rica (10 $12^{\prime} \mathrm{N}, 83^{\circ} 37^{\prime} \mathrm{W}$ ), específicamente en el lote 26 ("Donantes de Reforest The Tropics"). Se encuentra a $295 \mathrm{~m}$ de altitud, tiene una temperatura promedio anual de $25,1^{\circ} \mathrm{C}$ y una precipitación media anual de $3464 \mathrm{~mm}$. Los daños fueron detectados en una plantación mixta de 16 años y de 3,44 ha con las especies Vochysia guatemalensis, Araucaria hunsteinii e Hieronyma alchorneoides; esta última fue la especie afectada por la plaga. Al momento de la recolección de muestras la densidad era de 326 árboles por hectárea donde 130 corresponden a $H$. alchorneoides.

\section{Evaluación dasométrica}

Con base en las parcelas permanentes de muestreo (PPM), cuyos registros se encuentran hasta el 2014, se determinó el comportamiento del volumen de madera y el Incremento MedioAnual delvolumen (IMAvol) porhectárea.

\section{Diagnóstico}

Para obtener especímenes adultos, se colocaron seis trampas en seis árboles en los agujeros de entrada de mayor diámetro de las galerías elaboradas por las larvas y con secreciones. Estas trampas consistieron en cajas elaboradas con cedazo de metal de $0,4 \mathrm{~cm}$ adheridas al fuste mediante grapas superficiales. Las trampas se revisaron cada semana por dos meses.

El espécimen adulto obtenido fue colocado en un vial de vidrio con alcohol (90\%) y se llevó para su identificación al Instituto de Biodiversidad (InBIO), San José- Costa Rica.

\section{Descripción del daño}

Se cortaron tres árboles de H.alchorneoides que presentaban agujeros y secreciones en el fuste. Los fustes fueron cortados transversalmente a partir de los agujeros de mayor diámetro. Estas labores se realizaron en el campo y en el Centro de Investigación de Investigación Bosque Industria (CIIBI) del Instituto Tecnológico de Costa Rica (ITCR).

\section{Resultados}

\section{Diagnóstico}

Se obtuvo solamente un adulto, el cual fue identificado como Pantophthalmus sp. de la familia Pantophthalmidae y del orden Diptera. En Costa Rica se han registrado ocho especies del género Pantophthalmus (Zumbado, 2006); en este caso no se pudo identificar a nivel de especie, ya que el adulto se maltrató mucho en el cautiverio antes de ser recolectado, por el roce con el cedazo y la exposición a las precipitaciones. Se presenta a continuación la descripción de la larva con base en las observaciones de los especímenes recolectados. La descripción de los demás estados se hace con base en la información bibliográfica disponible.

La larva es de color azul-verdoso y puede llegar a medir 60 $\mathrm{mm}$ de largo y $15 \mathrm{~mm}$ de grosor (Figura 1.A). La cara dorsal del segmento protoráxico presenta franjas longitudinales color rojo oscuro separadas por franjas pálidas estrechas (Figura 1.B). La cápsula cefálica se encuentra fuertemente esclerotizada; el aparato bucal presenta una estructura córnea que se proyecta de la frente con un par de maxilas fuertes. El último segmento abdominal presenta una gran placa fuertemente esclerotizada color pardo oscuro con ocho protuberancias que cubre la abertura anal. 

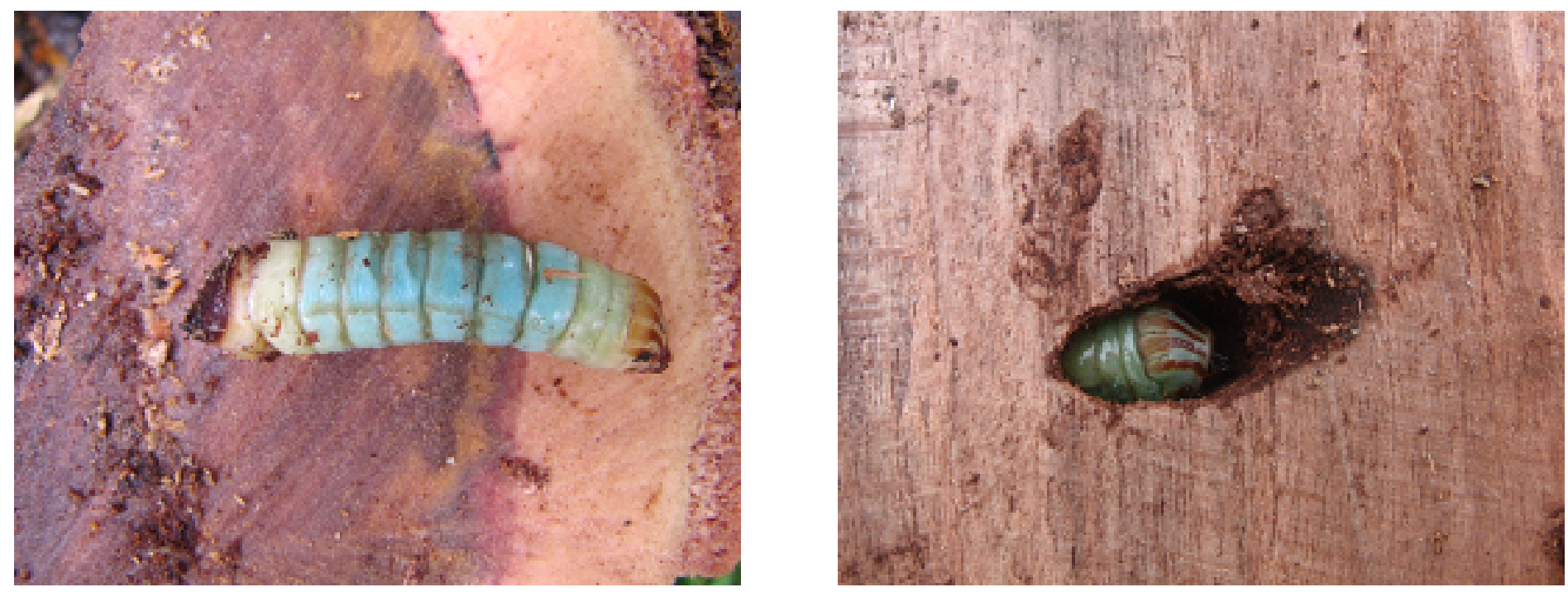

Figura 1. Larva de Pantophthalmus sp. en Hieronyma alchorneoides. A. vista dorsal; B. cara dorsal del segmento protoráxico. Limón, Costa Rica. Figure 1. Larva of Pantophthalmus sp. in Hieronyma alchorneoides. A. dorsal view; B. dorsal aspect of prothoracic segment. Limon, Costa Rica.

Los huevos tienen forma semejante a los granos de trigo y son colocados encima de la corteza o en grietas (Cibrián et al., 1995, Krivosheina, 2015). Las larvas pupan en las galerías; las pupas son color pardo, obtusamente truncadas en el extremo anterior del cuerpo y cubiertas de numerosos tubérculos esclerotizados; poco antes de que los adultos emerjan, las pupas salen parcialmente del agujero, quedando la exubia colgada de la perforación (Zumbado, 2006, Rapp, 2011, Krivoscheina, 2015).

Los adultos son moscas robustas de mediano a gran tamaño, miden entre 18-45 mm de longitud y se considera que alcanzan los tamaños más grandes de las moscas del mundo. La hembra es más grande que el macho; los ojos están muy separados en las hembras y unidos en los machos; las antenas tienen el tercer segmento anillado y generalmente son más largas y puntiagudas en los machos. El pronoto presenta dos líneas bien definidas descendentes negras y una tercera más tenue entre estas dos. Las alas tienen por lo general, un patrón de manchas oscuras y amarillas, presentan una celda discal amplia y las celdas $\mathrm{m} 3 \mathrm{y}$ anal son cerradas. Tiene patas negras, la tibia carece de espina apical y tienen tres almohadillas en el extremo (Val, 1976, Cibrián et al. 1995, Zumbado, 2006, Woodley, 2009).

\section{Descripción del daño}

Los árboles afectados presentan perforaciones circulares en el fuste de hasta $15 \mathrm{~mm}$ de diámetro (Figura 2.A). En la parte inferior de dichas perforaciones se observan exudaciones profusas color negruzcas en el fuste (Figura 2.B). De acuerdo a Rapp (2007), se pueden encontrar hasta 150 orificios en un solo fuste; sin embargo, muchos no se aprecian porque son inicialmente muy pequeños.
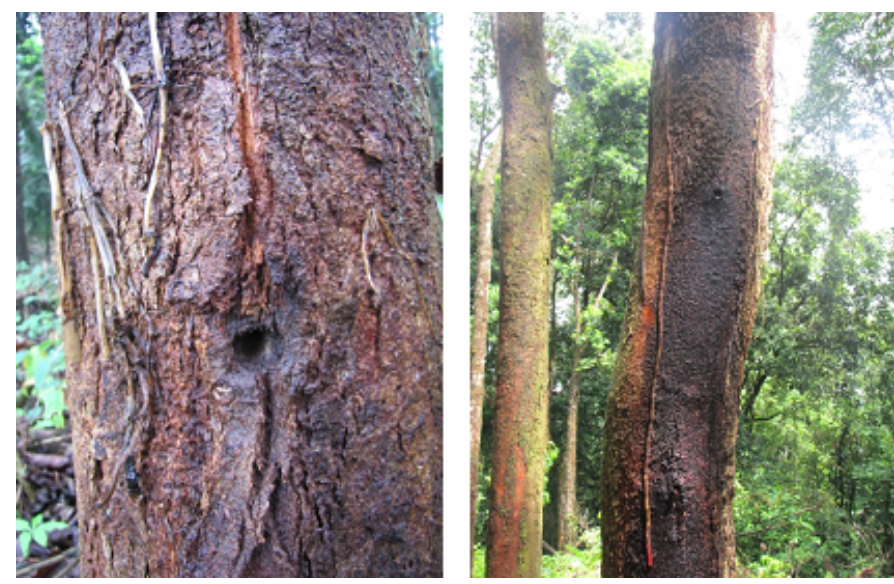

Figura 2. Daños visibles causados por Pantophthalmus sp. en el fuste de árboles en pie de Hieronyma alchorneoides. A. Perforación; B. Perforación y exudaciones sobre la corteza. Limón, Costa Rica.

Figure 2. Visible damage caused by Pantophthalmus sp. in the trunk of standing Hieronyma alchorneoides trees. A. Drilling; B. Drilling and exudates over crust. Limon, Costa Rica.

A partir de las perforaciones, las larvas forman galerías horizontales con un túnel central de hasta $16 \mathrm{~mm}$ de diámetro con varias ramificaciones secundarias. Las galerías abarcan tanto la albura como el duramen, pueden alcanzar hasta 16 $\mathrm{cm}$ de longitud y tienden a tener forma de arco (Figura 3).

La madera afectada por las galerías presenta también manchados oscuros, asociados con hongos y bacterias manchadoras (Figuras 3 y 4). De acuerdo a Cibrián et al., (1995), existe evidencia de una relación mutualista entre las larvas y hongos que provocan pudriciones líquidas de olor fétido, de la cual se alimentan las larvas. 

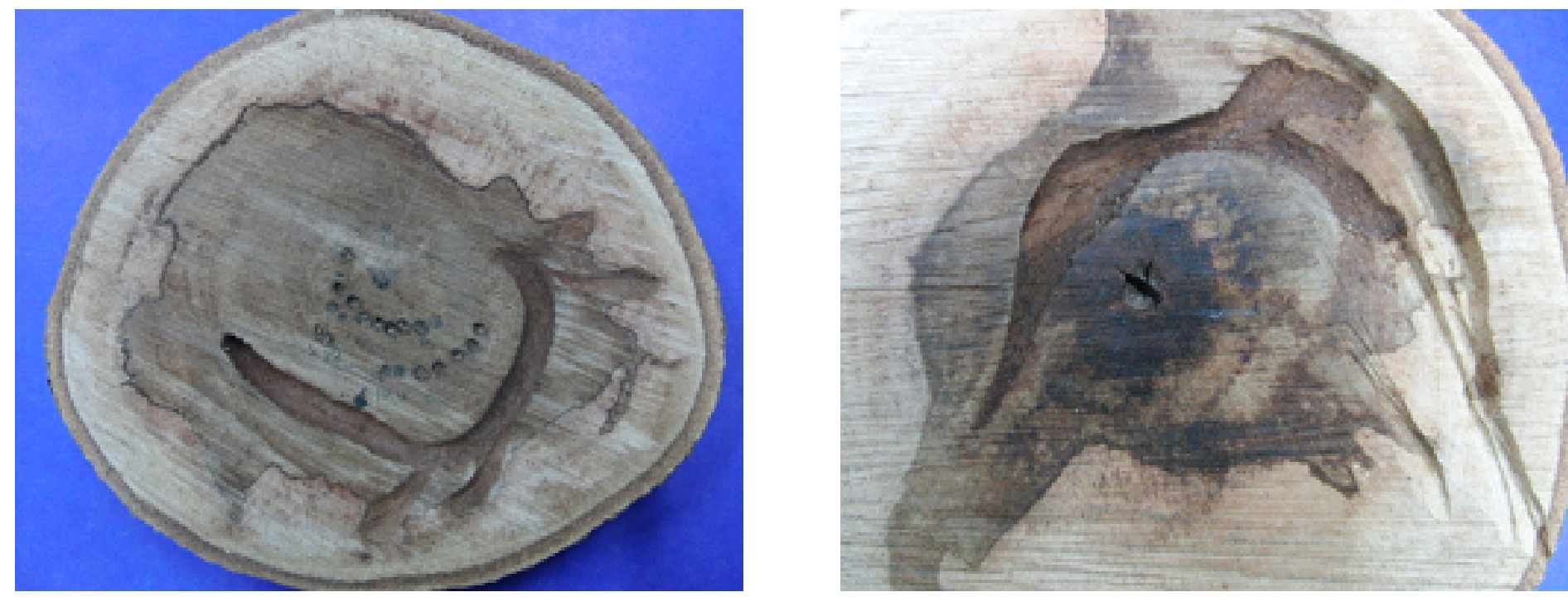

Figura 3. Galerías características formadas por Pantophthalmus sp. en el fuste de árboles en pie de Hieronyma alchorneoides. Limón, Costa Rica. Figure 3. Galleries features formed by Pantophthalmus sp. in the trunk of standing Hieronyma alchorneoides trees. Limon, Costa Rica.

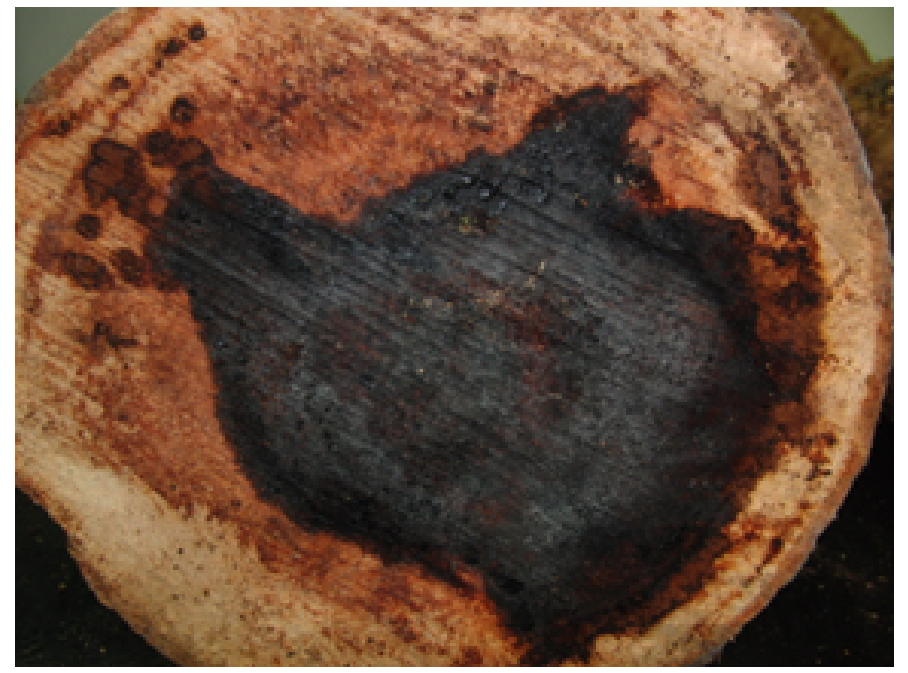

Figura 4. Pudriciones líquidas asociadas a los daños de Pantophthalmus sp. en árboles en pie de Hieronyma alchorneoides. Limón, Costa Rica.

Figure 4. Rotten liquids associated with Pantophthalmus sp. damage in standing Hieronyma alchorneoides trees. Limon, Costa Rica.

\section{Evaluación dasométrica}

El volumen máximo alcanzado por la plantación forestal afectada previo al segundo raleo en el año 15 fue de 337 $22,3 \mathrm{~m}^{3} /$ ha, correspondienteal Incremento Medio Anual del volumen (IMAVol) general de $22,3 \mathrm{~m}^{3} / \mathrm{ha} /$ año. Por especie, el IMAVol mayor es para $V$. guatemalensis (13,7 m³/ha/año), seguido por $\mathrm{H}$. alchorneoides (7,2 m³/ha/año) (Figura 5).

Zumbado (2006) y Rapp (2011) indicaron que generalmente las larvas de este género atacan árboles enfermos o recién muertos; sin embargo, los mismos

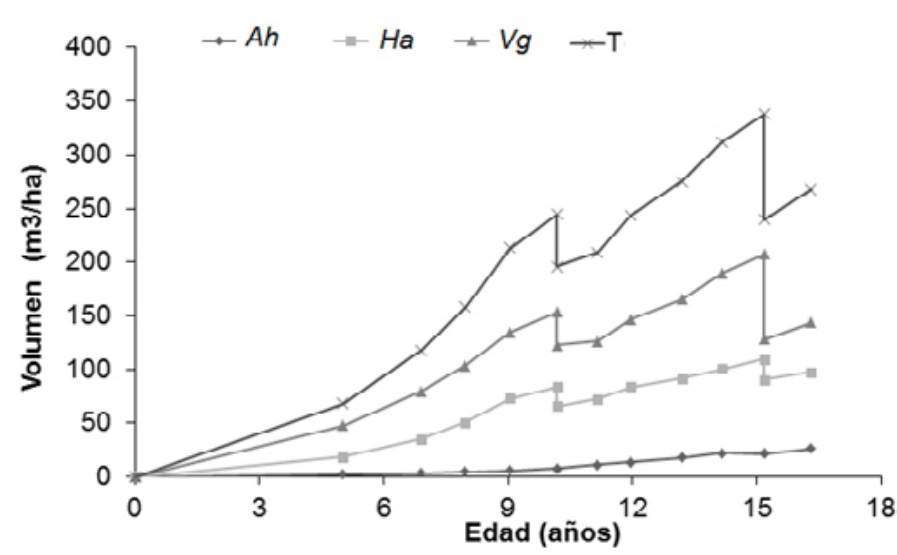

Leyenda: Ah: Araucaria hunsteinii, Ha: Hieronyma alchorneoides, Vg: Vochysia guatemalensis y T: total.

Figura 5. Volumen total por hectárea $\left(\mathrm{m}^{3} / \mathrm{ha}\right)$. Lote 26 (Donantes de Reforest The Tropics). Hacienda Las Delicias, Guácimo, Limón, Costa Rica.

Figure 5. Total volume per hectare (m3/ha). Lot 26 (Donor of Reforest The Tropics). Hacienda Las Delicias, Guácimo, Limón, Costa Rica.

autores y Cibrián et al., (1995), mencionan casos en que pueden atacar árboles aparentemente sanos y vigorosos. Krivosheina (2015) menciona que la condición de los árboles vivos infestados con Pantophthalmus no siempre se describe en publicaciones. En este caso en particular, se demuestra mediante la valoración dasométrica que dentro de la plantación afectada, el volumen de $H$. alchorneoides es $39 \%$ menor que el de $V$. guatemalensis, lo que indica que los individuos de $\mathrm{H}$. alchorneoides se encuentran suprimidos, con menor disponibilidad de luz y posiblemente de nutrimentos; esta condición podría tornarlos más susceptibles a este tipo de ataques. 


\section{Agradecimiento}

La autoría expresa su agradecimiento al ingeniero Rolando Camacho Herrera de la empresa Reforest The Tropics Inc. por toda su colaboración durante las labores de campo y por facilitar el uso de la información dasométrica.

\section{Referencias}

Abdelnour, A., Aguilar, M. \& Valverde, L. (2011). Micropropagación de Pilón (Hieronyma alchorneoides). Agronomía Costarricense, 35(2), 9-19.

Alice, F., Montagnini, F. \& Montero, M. (2004). Productividad en plantaciones puras y mixtas de especies forestales nativas en la Estación Biológica La Selva, Sarapiquí, Costa Rica. Agronomía Costarricense, 28(2), 61-71.

Cibrián, D., Méndez, T., Campos, R., Yates, H. \& Flores, J. (1995). Insectos forestales de México. México, Universidad Autónoma de Chapingo.

Krivosheina, N.P. (2015). The position of the family pantophthalmidae in the classification of the orthorrhaphous Brachycera (Diptera) based on larval characters. Entomological Review, 95 (1), 91-98

Montero M De los Santos Posadas, H. \& Kanninen, M. (2007). Hieronyma alchorneoides: ecología y silvicultura en Costa Rica. Serie Técnica. Informe Técnico/CATIE no. 354. Costa Rica, CATIE.

Rapp, M. (2007).The Immature Stages of Pantophthalmus planiventris (Wiedemann, 1821) (Diptera: Pantophthalmidae). Studia dipterol., 14, 27-36.

Rapp, M. (2011). The Immature Stages of Pantophthalmus bellardii (Bigot) and Late Immature Stages of Pantophthalmus kerteszianus (Enderlein) (Diptera: Pantophthalmidae), with a Review of Host-tree Relationships among the Family. Studia dipterol., 18 (1/2), 91-104.

Val, F.C.(1976). Systematicsand evolution of the Pantophthalmidae (Diptera, Brachycera). Arquivos de Zoologia, 27 (2), 51-174.

Woodley, N. (2009). Pantophthalmidae (Pantophthalmid flies). En Brown, B. (Ed.), Manual of Central American Diptera. National Reseach Council of Canada Volume 1, Canada, Research Press.

Zumbado, M. (2006). Dípteros de Costa Rica y la América Tropical/Diptera of Costa Rica and the New World tropics. Costa Rica. INBIO. 